\title{
Introduction to the special issue on computational optimization under uncertainty
}

\author{
Ronald Hochreiter • Georg Ch. Pflug
}

Published online: 16 December 2008

(C) Springer-Verlag 2008

\section{Introduction}

This special issue on Computational Optimization under Uncertainty was initiated during the 11th Conference on Stochastic Programming which was held at the University of Vienna in August 2007. A carefully selected range of papers has been chosen for publication in this issue. The aim is to provide an up-to-date overview of state-of-the-art stochastic programming techniques and applications. Most contributions consider the valuable but more challenging multi-stage case. Both contributions improving computational solutions of the optimization programs in general, as well as a set of specific applications from different management areas are covered.

The first part of this issue is dedicated to numerical solution frameworks and approaches for multi-stage stochastic programs. In the first paper, Holger Heitsch and Werner Römisch tackle the problem of calculating an optimal reduction of multi-stage scenarios trees to allow for more tractable computational solutions once the underlying scenario tree is huge. In contrast to the much simpler two-stage case of scenario (tree) reduction, the multi-stage case is handled by incorporating the filtration distance. An algorithm is presented, which aims at fulfilling predefined error tolerances. Jacek Gondzio and Andreas Grothey examine special structures of optimization problems, and present the implementation of the software library OOPS, which is an object oriented parallel solver using primal-dual interior point methods to exploit special matrix structures, which are often prevalent in multi-stage stochastic programming problems.

\footnotetext{
R. Hochreiter $(\varangle) \cdot$ G. Ch. Pflug

Department of Statistics and Decision Support Systems, University of Vienna,

Universitätsstraße 5, 1010 Vienna, Austria

e-mail: ronald.hochreiter@compmath.net

G. Ch. Pflug

e-mail: georg.pflug@univie.ac.at
} 
This specific implementation has been used to solve stochastic problems with more than 12 million scenarios on parallel computing systems using more than 1,000 processors in parallel. Jitka Dupačová, Marida Bertocchi, and Vittorio Moriggia apply contamination techniques to test the structure of multi-stage stochastic programs with multi-stage polyhedral risk objectives, and apply this procedure to a bond portfolio management problem.

In the second part, management decision optimization applications from the areas of Finance, Energy, and Transportation are presented. While Alois Geyer, Michael Hanke, and Alex Weissensteiner extend their previously successfully applied multistage stochastic asset management model to life-cycle investment problems, Markku Kallio and Antti Pirjetä develop a stochastic programming framework for option pricing based on marginal bid/ask price valuation. Both European and American style exercise structures are integrated using a double binary tree. Incentive options are valued considering hedging restrictions, and a large set of realistic constraints is included in the model. Miguel Carrion, Uwe Gotzes, and Rüdiger Schultz formulate a two-stage stochastic program including second-order stochastic dominance constraints to support an electricity retailer who wants to determine its forward contracting portfolio and the selling prices for potential clients. Finally, Francesca Maggioni, Michal Kaut, Luca Bertazzi study a single-sink transportation problem in which both the production capacity of suppliers, as well as the demand is stochastic. A two-stage and a multi-stage stochastic mixed integer linear programming model is formulated to solve this problem. Real data from the largest cement producer in Italy is used to analyze numerical results.

We want to thank the Editor-in-Chief Berc Rustem for providing the opportunity to publish this issue in Computational Management Science, and express our gratitude to all referees who supported the editorial process of this issue. 